\title{
EL ENFOQUE METACOGNITIVO EN LA EDUCACIÓN
}

\author{
The metacognitive aproach in education
}

por

\author{
$\underline{\text { Article record }}$ \\ HTML format
}

Narciso Barrero González ${ }^{\text {a }}$

\author{
$\underline{\text { Ficha del artículo }}$ \\ Formato HTML
}

La orientación metacognitiva de la educación se adopta con el ánimo de dar una respuesta en la práctica educativa a los problemas que los alumnos tienen para gestionar sus propios procesos cognitivos. Desde esta perspectiva la intención no es sólo instructiva sino que se pretende convertir al alumno en el protagonista y gestor ejecutivo de su crecimiento cognitivo y académico entrenándolo en estrategias consideradas relevantes por la investigación (Palincsar y Brown (1984; Brown, Palincsar y Armbruster, 1984; Palincsar y otros, 1991; Palincsar, Brown y Campione, 1993). Estos procedimientos posibilitan la supervisión y la mejora de las estrategias cognitivas siempre que la intervención se realice en contextos adecuados en los que se favorezca la mediación del profesor entre el alumno y los materiales objeto de aprendizaje.

Algunas de las estrategias consideradas más relevantes son la anticipación de hipótesis de significado, las auto-preguntas acerca de los procedimientos y la redacción de sumarios en el caso de comprensión de textos. Hasta el momento en que Palincsar y Brown deciden iniciar su investigación existían estudios sobre cada una de estas estrategias de forma aislada pero no considerándolas en su conjunto. Estos autores, desde una óptica pedagógica, integran las diversas estrategias en un mismo diseño de investigación educativa de forma secuencial. Posteriormente se ha generalizado la construcción de programas que integran gran número de estás estrategias con objeto de lograr resultados en la práctica educativa.

Las estrategias metacognitivas son muy resistentes a la enseñanza frontal y no pueden ser administradas mecánicamente. Cuando los programas metacognitivos se intentan impartir desde una óptica tradicional es posible que produzcan crisis entre el profesorado $\mathrm{y}$ el alumnado y tengan que abandonarse, esto se debe a que se trabaja sobre los procedimientos con contenidos móviles y flexibles (apariencia de libro vacío) en los hay pocas posibilidades de basar una enseñanza rutinaria y repetitiva que soslaye el discurso dialéctico del profesor y el alumno acerca de los procesos desarrollados.

En el desarrollo de programas metacognitivos los alumnos pueden tener algunas dificultades al tener que tomar la iniciativa y dirigir el curso de la acción, mediando el

\footnotetext{
a Facultad de Ciencias de la Educación (Universidad de Sevilla)
} 
profesor en esta circunstancia con las demostraciones previas necesarias y la práctica retroalimentada de forma constante. A medida que la intervención se desarrolla es común observar cómo los participantes comienzan a tomar la iniciativa y a implicarse personalmente en sus propios procesos se automonitorización (Collins, 1996).

El objetivo primordial de un diseño metacognitivo es que el alumno genere $a$ medida su propio programa. Esta pretensión, juzgada utópica por algunos, no lo es tanto si ofrecemos al alumno un módulo motivacional potente, la orientación necesaria, los materiales adecuados y el contexto más favorable.

Tanto el conjunto de contenido cognitivo acerca de la propia estructura de conocimiento, como los procesos metacognitivos de auto-observación de las propias estrategias, han sido objeto de una profusa investigación en el ámbito anglosajón.

En este monográfico presentamos algunos trabajos realizados en el contexto hispanoamericano en el que el enfoque metacognitivo se está abriendo paso en las últimas décadas. Hemos reunido una serie de trabajos que abordan el tema de la metacognición atendiendo a aspectos como la motivación del alumno, la evaluación del aprendizaje o las propias metodologías para "enseñar a pensar”. Completan el número, los artículos sobre la reelaboración y evaluación de un programa de estrategias metacognitivas para la mejora de la comprensión lectora, y sobre el efecto de un programa de desarrollo afectivo-emotivo a través de estrategias metacognitivas implementado con alumnos universitarios.

En el primero de los artículos incluidos en este número, Ugartetxea reflexiona sobre la posible relación existente entre metacognición y motivación. Entre otros, aspectos tales como la atribución de la causalidad, la localización del control o el establecimiento de expectativas de éxito centran la atención del autor, considerando que pueden estar condi- cionados por el conocimiento metacognitivo de los alumnos.

El trabajo de Martín Izard se centra en las metodología para la enseñanza de los procesos de pensamiento, arrancando del tratamiento que se hace de este tema desde la investigación, la práctica educativa y la legislación. Tras mostrar diferentes metodologías de "enseñar a pensar" (entrenamiento conductual cognitivo de Meichenbaum y metodología de la mediación de Feuerstein), presenta resultados de un estudio empírico que vendría a avalar la hipótesis de que estas metodologías facilitan la enseñanza de estrategias metacognitivas y la transferencia de las mismas.

El artículo de Barrero se aproxima al ámbito de la evaluación de la enseñanza desde la perspectiva de las estrategias metacognitivas. Desde este enfoque se estudia cómo los profesores median en que los alumnos automonitoricen y remodelen los propios procedimientos cognitivos, atendiendo la evaluación no sólo al producto de su mediación sino a todo un proceso auto-gestionado por el profesor de forma ejecutiva.

Repetto y colaboradores presentan el programa "Comprender y Aprender en el Aula", elaborado recientemente a partir de la revisión y modificación del conocido programa OMECOL, que constituyó el primero basado en estrategias cognitivas creado en nuestro país, en la década de los ochenta. En el artículo se presenta con detalle el diseño de evaluación previsto y se adelantan los resultados de la evaluación del programa en sí mismo, ya realizada.

Finalmente, el estudio empírico de Carvallo muestra la evaluación del "Programa de Desarrollo Humano" aplicado a alumnos universitarios de la Universidad Veracruzana (Méjico), a partir de un diseño de corte experimental con el que se pretendió comprobar que las puntuaciones en el Inventario de Configuración Psicológica (CPI) se incrementan en mayor medida en los alumnos del 
grupo experimental, a los que se aplicó el programa, que en los alumnos tomados como grupo control.

\section{REFERENCIAS}

BROWN, A.L., PALINCSAR, A.S. y ARMBRUSTER, B.B. (1984): Instructing comprehension-fostering activities in interactive learning situations. H. Mandl, N.L. Stein y T. Trabasso (Eds.), Learning and Comprehension of Text. Hillsdale, N.J.: Erlbaum.

COLLINS, N.D. (1996). Metacognition and Reading to Learn. ERIC Digest. Indiana: University.

PALINCSAR, A. y BROWN, A.L. (1984): Reciprocal teaching of comprehension- fostering and comprehension monitoring activities. Cognition and Instruction, 1, 117-75. (Tb. en J. Osborn, P. Wilson y R.C. Anderson (Eds.), Reading Education; Foundations for a Literate America. Lexington: M.A. Books).

PALINCSAR, A. y otros (1991). Examining the Context of Strategy Instruction. Remedial and Special Education (RASE); v12 n3 p43-53 May-Jun.

PALINCSAR, A., BROWN, A.L. y CAMPIONE, J. (1993). First grade dialogues for knowledge acquisition and use, contexts for learning. In E. Forman, N. Minick and C. Stone (Eds.), Sociocultural dynamics in children's development. N.York: Oxford U. Press.

\title{
ARTICLE RECORD / FICHA DEL ARTÍCULO
}

\begin{tabular}{|l|l|}
\hline $\begin{array}{l}\text { Reference / } \\
\text { Referencia }\end{array}$ & $\begin{array}{l}\text { Barrero González, N. (2001). El enfoque metacognitivo en la educación. RELIEVE, v. 7, n. 2. Consulta- } \\
\text { do en www.uv.es/RELIEVE/v7n2/RELIEVEv7n2_0.htm en (poner fecha). }\end{array}$ \\
\hline $\begin{array}{l}\text { Title / } \\
\text { Título }\end{array}$ & El enfoque metacognitivo en la educación \\
\hline $\begin{array}{l}\text { Authors / } \\
\text { Autores }\end{array}$ & Barrero González, Narciso \\
\hline $\begin{array}{l}\text { Review / } \\
\text { Revista }\end{array}$ & Revista ELectrónica de Investigación y EValuación Educativa (RELIEVE), v. 7, n.2 \\
\hline ISSN & $1134-4032$ \\
\hline $\begin{array}{l}\text { Institution / } \\
\text { Institución }\end{array}$ & Universidad de Sevilla (España) \\
\hline $\begin{array}{l}\text { Publication } \\
\text { site / } \\
\text { Dirección }\end{array}$ & http://www.uv.es/RELIEVE \\
\hline $\begin{array}{l}\text { Language / } \\
\text { Idioma }\end{array}$ & Spanish (Title, abstract and keywords in English ) \\
\hline
\end{tabular}

\section{Revista ELectrónica de Investigación y EValuación Educativa (RELIEVE)}

\author{
[ ISSN: 1134-4032 ]
}

\begin{abstract}
(C) Copyright, RELIEVE. Reproduction and distribution of this articles it is authorized if the content is no modified and their origin is indicated (RELIEVE Journal, volume, number and electronic address of the document).

(C) Copyright, RELIEVE. Se autoriza la reproducción y distribución de este artículo siempre que no se modifique el contenido y se indique su origen (RELIEVE, volumen, número y dirección electrónica del documento).
\end{abstract}

